\title{
Thermoreflectance Imaging of Semiconductor Lasers With a Numerical Thermal Model
}

\author{
Robert McKenna ${ }^{\circledR}$, Simon Corbett, Sepideh T. Naimi, Dovydas Mickus ${ }^{\circledR}$, David McCloskey, \\ and John F. Donegan ${ }^{\circledR}$, Senior Member, IEEE
}

\begin{abstract}
High resolution surface temperature maps of a slotted surface grating laser operating at approx. $1550 \mathrm{~nm}$ are obtained by means of CCD-thermoreflectance imaging. The resolution is such that the temperature of the $2 \mu \mathrm{m}$ wide ridge can be determined. Experimental temperature profiles along the ridge and in the lateral direction are provided. A 2D numerical model is developed to simulate the steady state thermal distributions in the laser. There is good agreement between the experiment and simulation. This technique allows for high-resolution imaging and will be useful where hot spots occur on laser devices.
\end{abstract}

Index Terms-Semiconductor lasers, thermoreflectance imaging.

\section{INTRODUCTION}

EMICONDUCTOR lasers play an important role in a di$\checkmark$ verse range of applications including optical communications, gas sensing, and light detection and ranging (LIDAR), and are subject to stringent performance requirements. The temperature distributions present in the laser during operation are of great interest to device designers as large temperature excursions and hot spots can lead to poor device performance and reduced lifespan. In addition, the dependence of both the material bandgap and the refractive index on temperature leads to a strong temperature dependence of the emission wavelength. Thermal modelling is an important step in device design, and numerous models have led to detailed analysis of temperature distributions in simulated devices [1]-[3]. High resolution experimental investigation of thermal effects in semiconductor lasers have been done with a number of techniques including IR-SNOM [4], [5] and CCD thermoreflectance imaging (CCDTR). CCD-TR is a well-established technique to measure the

Manuscript received April 1, 2021; revised August 23, 2021; accepted September 2, 2021. Date of publication September 8, 2021; date of current version September 24, 2021. This work was supported in part by Science Foundation Ireland under Project 15/IA/2854. (Corresponding author: Robert McKenna.)

Robert McKenna, Simon Corbett, Sepideh T. Naimi, and Dovydas Mickus are with the School of Physics, Trinity College Dublin, D02 PN40 Dublin, Ireland (e-mail: mckennr2@tcd.ie; corbetsi@tcd.ie; sepideh.naimi@tcd.ie; mickusd@tcd.ie).

David McCloskey and John F. Donegan are with the School of Physics, Trinity College Dublin, D02 PN40 Dublin, Ireland, and also with the Future Networks and Communications (CONNECT), Centre for Research on Adaptive Nanostructures and Nanodevices, (CRANN) and Advanced Materials and BioEngineering Centre (AMBER), Trinity College Dublin, D02 PN40 Dublin, Ireland (e-mail: mccloskd@tcd.ie; jdonegan@tcd.ie).

Color versions of one or more figures in this article are available at https://doi.org/10.1109/JSTQE.2021.3110884.

Digital Object Identifier 10.1109/JSTQE.2021.3110884 (a)

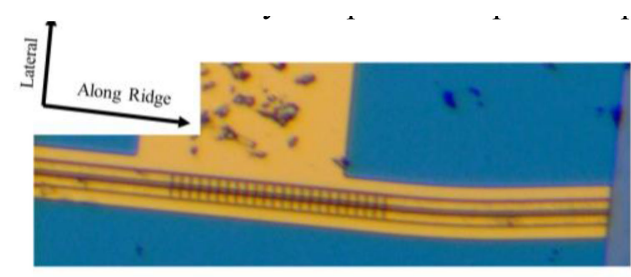

(b)
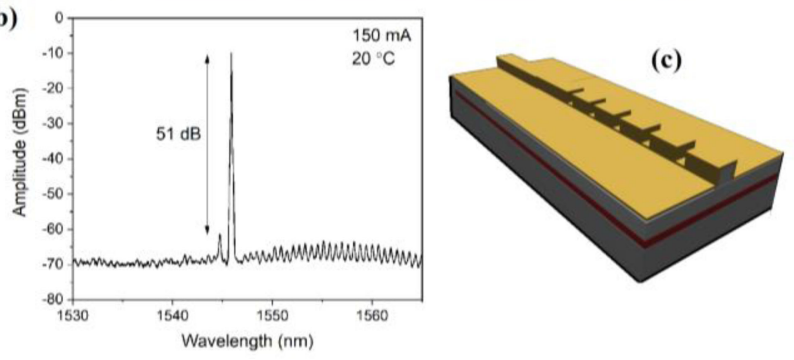

Fig. 1. (a) Optical microscope image. (b) Lasing spectrum of the laser diode at $150 \mathrm{~mA}$ bias current and $20^{\circ} \mathrm{C}$ heatsink temperature. (c) 3D representation of laser showing slots formed on the ridge. The slots forming the grating can be seen in the centre part of the image in (a).

surface temperature of electronic and optoelectronic devices and has been used for the thermal characterization of high power lasers [6], VCSELs [6]-[9], quantum dot lasers and quantum cascade lasers (QCLs) [10]-[12]. In this work we use CCD-TR imaging to image the surface temperature of a single frequency single-mode slotted semiconductor laser operating at approximately $1550 \mathrm{~nm}$. Preliminary measurements of the single and multi-section laser by our group are found in [13]. In addition, we show the results from a $2 \mathrm{D}$ model of the thermal behavior of the laser. The resolution of the imaging system is such that the temperature of the $2 \mu \mathrm{m}$ wide laser ridge can be determined.

\section{DeVICE Structure}

An image of the laser investigated in this work is shown in Fig. 1. The laser consists of a $2 \mu \mathrm{m}$ wide, $1.85 \mu \mathrm{m}$ tall ridge that runs along the length of the device. The laser cavity is formed by the reflections provided by the rear facet and the high-order grating in the center of the device. The remainder of the ridge waveguide from the grating to the front facet is external to the laser cavity and provides optical amplification.

The ridge waveguide is curved at $7^{\circ}$ to the front facet to reduce reflections from the front facet and to prevent the formation of another cavity. The ridge is composed of p-doped InP and 
sits above an active region comprised of 5 AlGaInAs compressively strained quantum wells which provide optical gain. The high-order grating is formed by etching rectangular slots 1 $\mu \mathrm{m}$ in width into the ridge and provides wavelength selective reflection. The use of a high-order surface grating was chosen rather than a buried grating because it simplifies the fabrication by avoiding the need for material regrowth and allows the use of photolithography, although in the case of the devices studied here electron beam lithography was used due to the lack of a stepper at the foundry. A $280 \mathrm{~nm}$ layer of Au covers the ridge, to allow current injection. The laser is bonded to an aluminum nitride subcarrier with is positioned on a copper heatsink that is temperature controlled with a thermoelectric module. Thermal compound with a thermal conductivity of $0.65 \mathrm{Wm}^{-1} \mathrm{~K}^{-1}$ is applied between the underside of the subcarrier and heatsink to ensure a good thermal contact. An optical spectrum of the device operating at $150 \mathrm{~mA}$ injection current with the heatsink temperature maintained at $20^{\circ} \mathrm{C}$ is shown in Fig. 1, showing good single mode performance with the side mode suppression ratio (SMSR) of $51 \mathrm{~dB}$. Modelling and characterization of slotted laser diodes can be found in [14]-[17].

\section{2D THERMAL MODEL}

The flow of heat through a solid is governed by the heat conduction equation, given by

$$
K \nabla^{2} T+Q=\rho C \frac{\partial T}{d t}
$$

where $T$ is the temperature, $K$ is the thermal conductivity, $\rho$ is the density, $C$ is the specific heat capacity, and $Q$ is the heat generation in the solid. In steady state the time derivative is zero, yielding,

$$
K \nabla^{2} T=-Q
$$

We assume that heat generation in the laser is due only to the Joule heating due to carrier flow, such that the heating per unit volume of a region of the laser structure is given by,

$$
Q=j^{2} \rho
$$

where $j$ is the current density and $\rho$ is the resistivity of the material, and to optical absorption in the active region, for which take the approach used by Wang and Yu [18], wherein the heating is given by

$$
Q=\frac{V_{j}\left(1-n_{s p} f_{s p}\right)}{d_{a}}\left(j_{t h}+\left(j-j_{t h}\right)\left(1-n_{i}\right)\right)
$$

where $V_{j}$ is the active region junction voltage, $n_{s p}$ is the quantum efficiency of spontaneous emission, $f_{s p}$ is the fraction of spontaneous emission absorbed by the cladding layers, $d_{a}$ is the active region thickness, $n_{i}$ is the internal quantum efficiency of stimulated emission and $j_{t h}$ is the threshold current density. Equations (3) and (4) are sufficient to account for the heat generation within the laser structure, however another term must be included to take account of the heat generated from the injection of holes from the metal into semiconductor at the upper contact. In our device, the upper $200 \mathrm{~nm}$ of the ridge is composed of a highly doped $\mathrm{p}+\mathrm{InGaAs}$ region to achieve ohmic contacts and minimize contact resistance. However, by scanning the voltage along the laser facet Kuntze et al. have shown that while minimizing metal-semiconductor contact resistance this approach generates a large voltage drop and thus heat generation at the $\mathrm{p}+-\mathrm{p}$ interface [19]. This can be characterized by another contact resistance, which they found to be $6 \times 10^{-9} \Omega \mathrm{m}^{2}$. Here we model this heat source with a $400 \mathrm{~nm}$ tall region at the upper part of the ridge with the heat generation per unit volume given by

$$
Q=j^{2} \frac{\rho_{c}}{d_{c}}
$$

where $\rho_{c}$ is the contact resistance and $d_{c}$ is the thickness of the contact region.

In order to determine the spatially resolved heat generation, the model must determine the current flow through the device structure. The current density is calculated by

$$
j=\sigma E+j_{e}
$$

where $\sigma$ is the electrical conductivity of the material, $E$ is the electric field and $j_{e}$ are externally generated current densities. The electric field is given by

$$
E=-\nabla \phi
$$

where $\phi$ is the electric potential. The electrical conductivities are given by

$$
n e \mu_{e}
$$

for the substrate region, and

$$
p e \mu_{h}
$$

where $n$ and $p$ are the electron and hole densities, $e$ is the charge on an electron, and $\mu_{e}$ and $\mu_{h}$ are the electron and hole mobilities respectively. The substrate doping level is $3 \times 10^{18}$ $\mathrm{cm}^{-3}$. The electron mobility of InP at this carrier concentration level was found by Sotoodeh et al. to be $2000 \mathrm{~cm}^{2} \mathrm{~V}^{-1} \mathrm{~s}^{-1}$ at room temperature leading to a substrate electrical conductivity of $1.1 \times 10^{5} \mathrm{~S} \mathrm{~m}^{-1}$. The cladding consists of a number or layers of various doping levels and the resulting cladding doping level is found by taking a weighted average of these layers, coming out to $1 \times 10^{18} \mathrm{~cm}^{-3}$. The hole mobility of InP at room temperature at this doping level was found by Sotoodeh et al. to be $70 \mathrm{~cm}^{2} \mathrm{~V}^{-1}$ $\mathrm{s}^{-1}$ leading to a cladding electrical conductivity of $1.4 \times 10^{3} \mathrm{~S}$ $\mathrm{m}^{-1}$ [20].

To reduce the computational requirements of the simulation a $2 \mathrm{D}$ model of the laser cross section was chosen. This model relies on the assumption that the temperature profile is reasonably constant along the length of the laser cavity. Thermoreflectance images of the surface shown in Section $\mathrm{V}$ indicate that this assumption is reasonably valid. The $2 \mathrm{D}$ geometry is shown in Fig. 2. The epitaxial structure of the laser is simplified into three sections, the substrate, active region, and cladding. The substrate is n-type $\mathrm{InP}$, the cladding is p-type $\mathrm{InP}$, and the active region consists of the quantum wells. Above the cladding lies a $300 \mathrm{~nm}$ oxide layer and a $280 \mathrm{~nm}$ gold layer. The substrate sits on $20 \mu \mathrm{m}$ of solder and a $0.5 \mathrm{~mm}$ thick aluminum nitride sub mount. The model parameters are listed in Table I. The material constants 


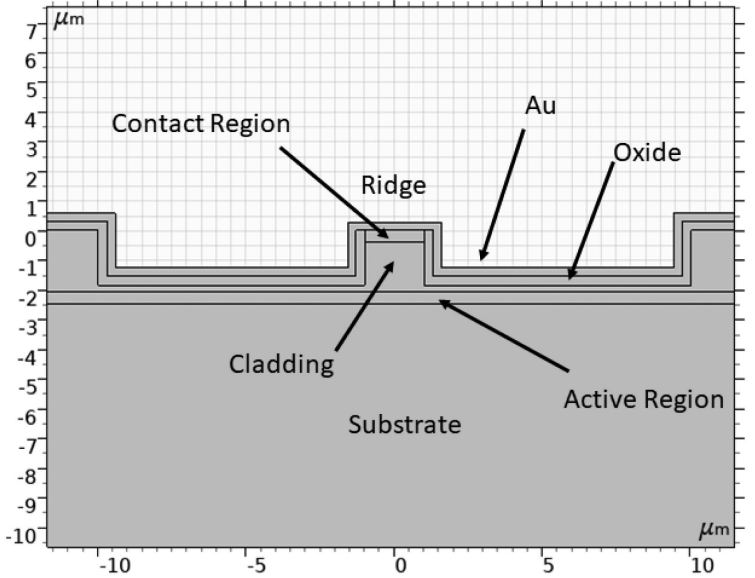

Fig. 2. Geometry of the numerical model.

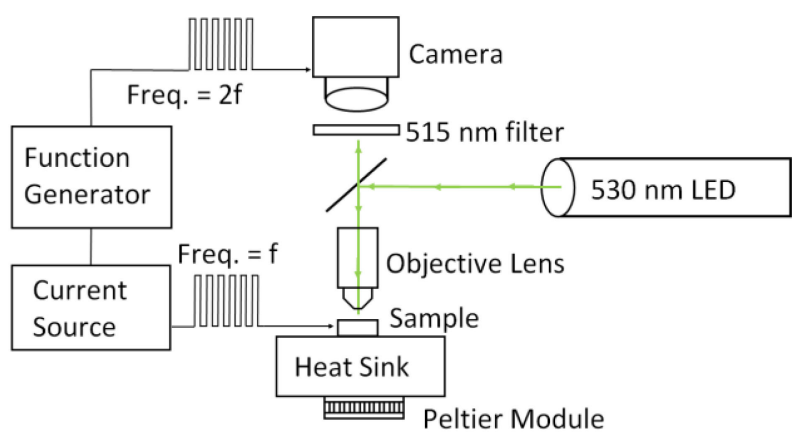

Fig. 3. Experimental setup to measure the CCD-TR images.

TABLE I

MODEL PARAMETERS

\begin{tabular}{llll}
\hline \hline Parameter & $\begin{array}{l}\text { Symbol } \\
\text { (unit) }\end{array}$ & Value & Reference \\
\hline Ridge Width & $w_{r}(\mu \mathrm{m})$ & 2 & \\
Ridge Height & $d_{r}(\mu \mathrm{m})$ & 1.8 & \\
Active Region thickness & $d_{a}(\mu \mathrm{m})$ & 0.4 & \\
Substrate thickness & $d_{s u b}(\mu \mathrm{m})$ & 120 & \\
Gold thickness & $d_{g}(\mu \mathrm{m})$ & 0.3 & \\
Oxide thickness & $d_{o x}(\mu \mathrm{m})$ & 0.28 & \\
Chip width & $w_{c}(\mu \mathrm{m})$ & 3500 & \\
Subcarrier width & $w_{s c}(\mu \mathrm{m})$ & 10000 & \\
Subcarrier thickness & $d_{s c}(\mu \mathrm{m})$ & 500 & \\
Solder thickness & $d_{s o l}(\mu \mathrm{m})$ & 20 & \\
Threshold current & $I_{t h}(\mathrm{~mA})$ & 50 & \\
Junction voltage & $V_{j}(\mathrm{~V})$ & 0.8 & \\
Internal quantum & $n_{i}$ & 0.8 & \\
Efficiency & & & \\
Quantum efficiency of & $n_{s p}$ & 0.4 & \\
Spontaneous emission & & & \\
Fraction of spontaneous & \multicolumn{1}{c}{0.59} \\
emission absorbed & $f_{s p}$ & 0.59 & \\
\hline \hline
\end{tabular}

are listed in Table II. The simulation is implemented with a finite element method (FEM) on the COMSOL Multiphysics software.

\section{CCD-THERMOREFLECTANCE}

CCD-Thermoreflectance imaging is a technique that allows high resolution thermal imaging of heated microelectronic and photonic devices. It has been used extensively to measure temperature distributions in integrated circuits and optoelectronic devices [3], [6]-[12], [21]-[26]. It makes use of the temperature dependence of the reflectance of the sample to measure changes in temperature. As the wavelengths utilized are in the visible region, the spatial resolution of the technique is much improved compared to IR thermography, which is limited to wavelengths in the 3-10 $\mu \mathrm{m}$ region [27]. As such, sub-micron resolution can be achieved. The temperature change can be determined from the reflectance change by

$$
\Delta T=\frac{1}{\kappa} \frac{\Delta R}{R}
$$

where $\Delta R$ is the change in reflectance and $\kappa$ is the thermoreflectance coefficient, which is dependent on the sample material, illumination wavelength and the numerical aperture of the imaging system. In this work, Au is chosen as the thermoreflectance material as it coats the upper surface of the laser. An illumination wavelength of $514.5 \mathrm{~nm}$ and a 20x objective lens with a numerical aperture of 0.4 are chosen. The thermoreflectance coefficient can vary by over two orders of magnitude and so must be calibrated. This is done by comparing the reflectance change of a thin Au stripe, which is heated by current injection, to the change in resistivity of the stripe. Although the resistivity of a metal is dependent on sample geometry, the temperature dependence of the resistivity is not. As such it can be used as a temperature probe. By this method we found $\kappa=-1.4 \times 10^{-4} \mathrm{~K}^{-1}$

The thermoreflectance effect is weak and requires a lock-in technique. Here we utilize a homodyne technique wherein the sample is heated by current injection at a frequency $f$ and the CCD camera is triggered with a synchronized signal at a frequency $2 f$, capturing one hot image and one cold image each heating cycle. In effect, each pixel of the CCD camera acts as a lock-in detector. Here, we use a camera frequency, $f$, of $25 \mathrm{~Hz}$ and take a total of 10000 images, yielding a measurement time of 400 seconds. A schematic of the setup is displayed in the Fig. 2. A $530 \mathrm{~nm}$ Thorlabs M 530L4 LED with a maximum output power of approximately $400 \mathrm{~mW}$, a Thorlabs $4070 \mathrm{M}$ CCD camera, a GW Instek AFG-2225 Arbitrary Function Generator, and a ILX-Lightwave LDC-3220 current source were used.

\section{REsULts}

Thermoreflectance images of the laser biased with 100, 150, 200 and $250 \mathrm{~mA}$ are shown in Fig. 4. The region from the rear HR facet to the middle of the grating are shown. The profiles along the top of the ridge are shown in Fig. 5(top) and the profile lateral to the direction of the ridge, averaged along the length of the unslotted region, are shown in Fig. 5(bottom). Care must be taken when interpreting these results as height steps in the surface topology can cause large reflectance changes that dominate over temperature induced change, leading to false temperature excursions. The height steps at the ridge edge and the trench walls are located in the shaded regions of Fig. 5(bottom). In addition, the topmost plot in this figure shows the image intensity of the monotone microscope image, scaled so as to fit on the plot, allowing easy identification of the ridge. The large undulations 
TABLE II

MATERIAL CONSTANTS

\begin{tabular}{llllll}
\hline \hline Material & $\begin{array}{l}\text { Electrical } \\
\text { Conductivity }\left(\mathrm{S} \mathrm{m}^{-1}\right)\end{array}$ & $\begin{array}{l}\text { Thermal Conductivity } \\
\left(\mathrm{W} \mathrm{m}^{-1} \mathrm{~K}^{-1}\right)\end{array}$ & Relative permittivity & $\begin{array}{l}\text { Density } \\
\left(\mathrm{kg} \mathrm{m}^{-3}\right)\end{array}$ & $\begin{array}{l}\text { Heat Capacity } \\
\left(\mathrm{J} \mathrm{K}^{-1} \mathrm{~kg}^{-1}\right)\end{array}$ \\
\hline $\mathrm{Au}$ & $4.56 \times 10^{7}$ & 317 & 1 & $1.93 \times 10^{4}$ & 129 \\
$\mathrm{SiO}$ & 0 & 1.4 & 4.2 & $2.2 \times 10^{3}$ & 730 \\
$\mathrm{p}-\mathrm{InP}$ & $1.4 \times 10^{3}$ & 68 & 12.5 & $4.81 \times 10^{3}$ & 180 \\
Active & $1.0 \times 10^{4}$ & 5 & 12.5 & $4.81 \times 10^{3}$ & 250 \\
Region & & & & & \\
$\mathrm{n}-\mathrm{InP}$ & $1.1 \times 10^{5}$ & 68 & 12.5 & $4.81 \times 10^{3}$ & 180 \\
Solder & $1 \times 10^{6}$ & 20 & 1 & $8.6 \times 10^{3}$ & 1670 \\
AlN & $1 \times 10^{6}$ & 180 & 1 & $3.26 \times 10^{3}$ & 1000 \\
\hline \hline
\end{tabular}

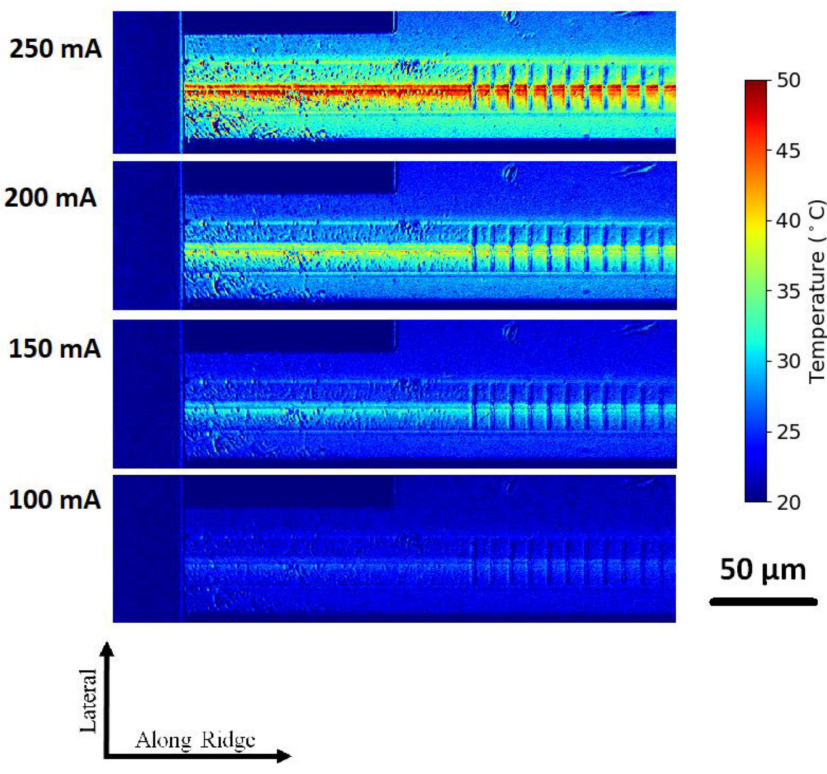

Fig. 4. Thermoreflectance images of the laser under various bias conditions. The heatsink is maintained at $20^{\circ} \mathrm{C}$.

in temperature seen on the right hand side of Fig. 5(top) result for the presence of the slots on the ridge and are artifacts of the measurement system. It is clear that the temperature profile along the length of the ridge remains approximately constant, with no discernable temperature gradient along the length of the laser ridge. On the other hand, in Fig. 5(bottom) there is a strong temperature gradient decaying away from the ridge, providing justification for the use of a 2D numerical model with the simulation plane in the direction perpendicular to the ridge, which greatly reduces the computational requirement over a 3D model.

Fig. 6 shows the simulated temperature of the laser operating at $200 \mathrm{~mA}$. The hottest point on the laser is at the top of the ridge with the temperature decaying both down into the structure and laterally, although the ridge temperature is reasonably constant throughout the ridge, at around $35^{\circ} \mathrm{C}$. The temperature decreases rapidly below the active region. The average ridge temperature in the unslotted region is plotted against the simulated average top of ridge temperature in Fig. 7. The use of the contact resistance measured in Kuntze et al. leads to a somewhat higher temperature (red curve) than measured by thermoreflectance. Reduction of the contact resistance to $4 \times 10^{-9} \Omega \mathrm{m}^{2}$ results an improved agreement between experiment and simulation,
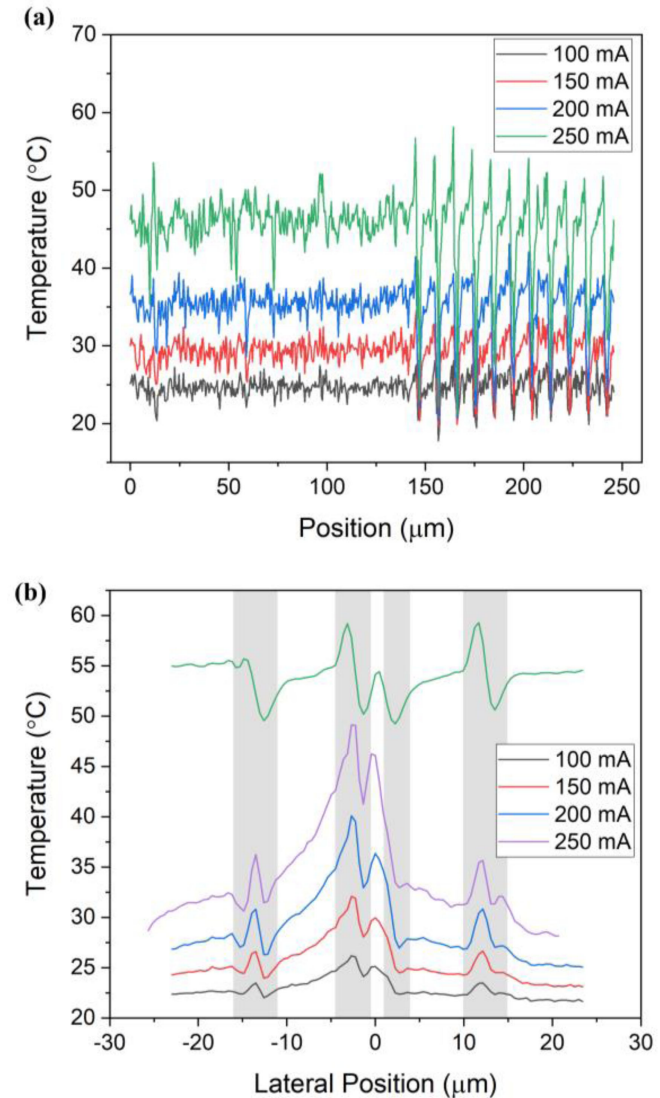

Fig. 5. Thermoreflectance temperature profiles in the direction (a) along the length of the ridge and (b) lateral to the ridge, for bias currents from $100 \mathrm{~mA}$ to $250 \mathrm{~mA}$. The heatsink is maintained at $20{ }^{\circ} \mathrm{C}$. The uppermost profile in (b) shows the pixel intensity of the microscope scaled to fit on the graph, to identify the ridge.

and is used in the remainder of this work. The average ridge temperature can also be deduced by considering the wavelength drift, which increases at a rate of $0.1 \mathrm{~nm}^{\circ} \mathrm{C}^{-1}$. The wavelength data shows slightly higher temperature than obtained through thermoreflectance.

Optical emission from the laser front facet is collected with a lensed fiber brought to the edge of the facet. The optical signal is split with a 90:10 splitter, with $90 \%$ sent to an Aglient 86140B optical spectrum analyser and $10 \%$ to a photodiode to aid coupling. Unfortunately, the thermoreflectance setup is not built with a mechanism to collect optical signal from the laser output, and as such the sample had to be moved to a new 


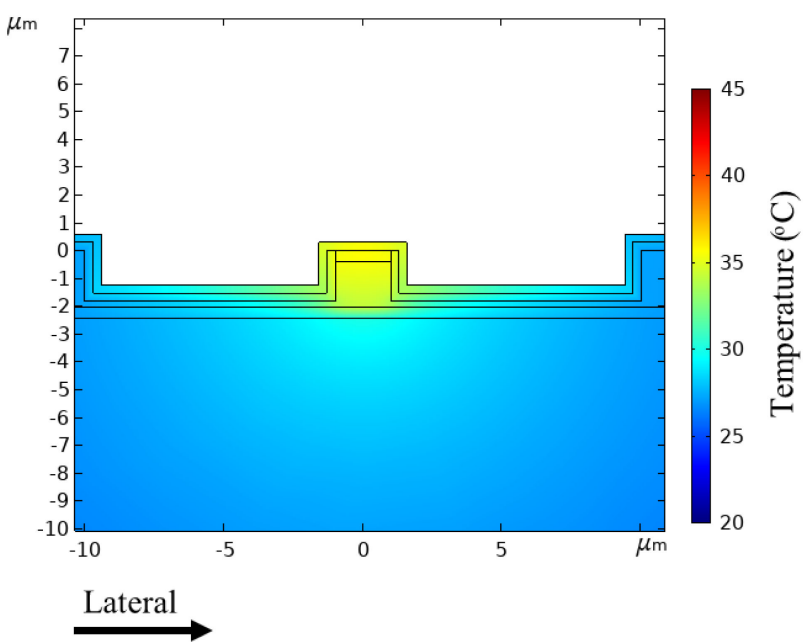

Fig. 6. Simulated temperature of the laser biased at $200 \mathrm{~mA}$. The top of the ridge shows the highest temperature.

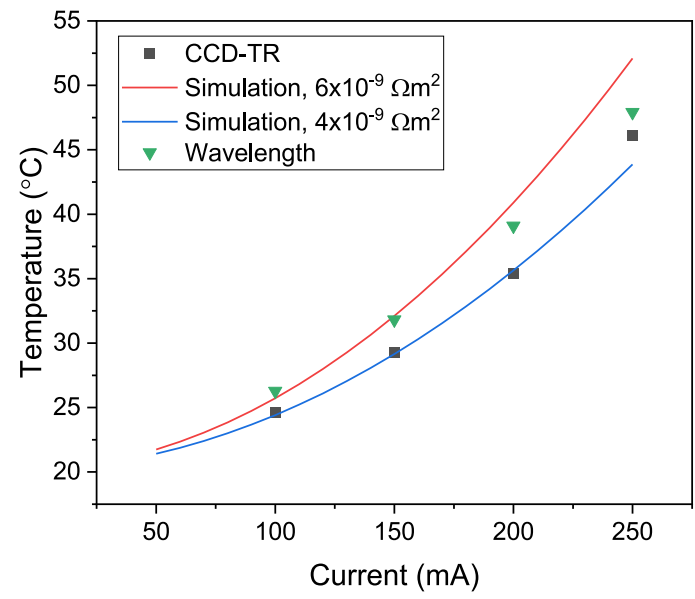

Fig. 7. Comparison between the experimental and simulated average temperature along the unslotted portion of the laser ridge. The simulated curves differ in choice of contact resistance, $6 \times 10^{-9}$ (red) and $4 \times 10^{-9}$ (blue) $\Omega \mathrm{m}^{2}$. Also shown is the temperature as determined by the lasing wavelength drift.

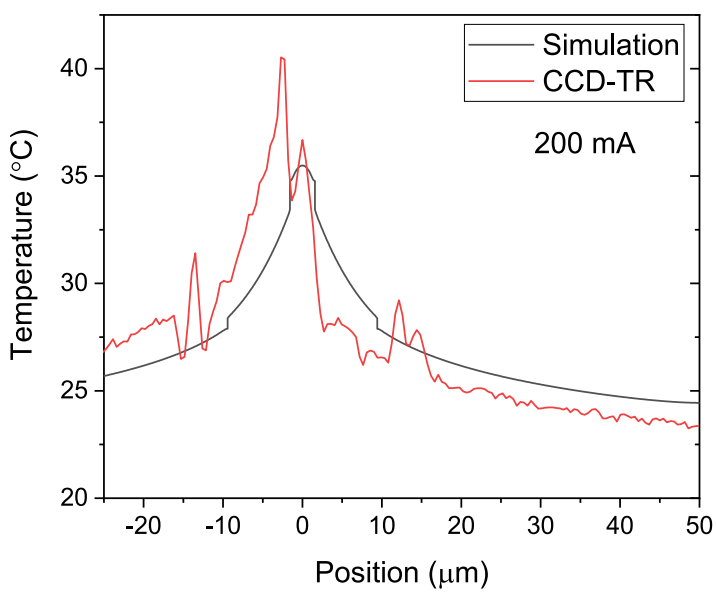

Fig. 8. Comparison between the experimental and simulated lateral temperature profiles at biased at $200 \mathrm{~mA}$. setup for wavelength measurement. Discrepancy between the thermal contact between the submount and heatsink for the two setups may account for this difference. A comparison of the lateral thermoreflectance temperature profile and the simulation temperature along the upper surface of the Au layer biased at $200 \mathrm{~mA}$ is shown in Fig. 8. There is considerable disagreement within $\pm 15 \mu \mathrm{m}$ of the ridge, however this is likely due to artefacts of the thermoreflectance technique in the vicinity of the ridge. Agreement picks up again from $15 \mu \mathrm{m}$, however the simulation shows a $1{ }^{\circ} \mathrm{C}$ higher temperature than the experiment though the fall-off in both graphs is similar.

\section{CONCLUSION}

In this work we demonstrate the use of thermoreflectance imaging as a key tool in determining the thermal profile of laser diodes and as complementary experimental feedback for the numerical thermal modelling of semiconductor lasers. We developed a numerical 2D thermal model which calculates the temperature rise in the laser due to self-heating. The temperature rise on the laser ridge as calculated by the model agrees reasonably well with that found from experimental imaging. Also, the temperature decay in the direction lateral to the ridge also shows similar agreement, however image noise makes accurate temperature determination difficult in the regions immediately surrounding the ridge. These findings justify the use of $s 2 \mathrm{D}$ model over more computationally expensive 3D models.

We found that the region of highest temperature to be the area around the metal-semiconductor junction and that the high temperature region is largely confined to the p-type ridge. The agreement between experiment and modelling of the lateral thermal decay is important for the efficient placement of additional devices for the case of laser arrays.

In the coming decade, the requirement for much improved energy efficiency in photonic devices will be apparent, even down to the device level. The present pandemic has shown the need for optical communications. This need will grow strongly in the coming decade. The CCD-TR imaging technique and the associated thermal modelling will be essential to build the next generation of lasers with such improved efficiency.

\section{REFERENCES}

[1] X. Li and W-P. Huang, "Simulation of DFB semiconductor lasers incorporating thermal effects," IEEE J. Quantum Electron., vol. 31, no. 10, pp. 1848-1855, Oct. 1995.

[2] S. Bozorgui, A. Destrez, and Z. Toffano, "An analysis of thermal effects and its influence on the laser dynamics in three-electrode DBR lasers," in Proc. EDMO, 1996, pp. 108-113, doi: 10.1109/EDMO.1996.575811.

[3] P. Kozodoy et al., "Thermal effects in monolithically integrated tunable laser transmitters," IEEE Trans. Compon. Packag. Technol., vol. 28, no. 4, pp. 651-657, Dec. 2005.

[4] B. - J. Pandey et al., "IR-SNOM on a fork: Infrared scanning near-field optical microscopy for thermal profiling of quantum cascade lasers," in Quantum Sensing and Nano Electronics and Photonics XVII, Jan. 2020, vol. 11288, p. 112881Q, doi: 10.1117/12.2543849.

[5] T. B. Daunis et al., "Infrared scanning near-field optical microscopy (IR-SNOM) for thermal profiling of quantum cascade lasers," in Optical Fibers and Sensors for Medical Diagnostics, Treatment and Environmental Applications XXI, Bellingham, WA, USA: SPIE, Mar. 2021. 
[6] M. Brunner, K. Gulden, R. Hövel, M. Moser, and M. Ilegems, "Thermal lensing effects in small oxide confined vertical-cavity surfaceemitting lasers," Appl. Phys. Lett., vol. 76, no. 1, pp. 7-9, Jan. 2000, doi: $10.1063 / 1.125638$

[7] M. Dabbicco, V. Spagnolo, M. Ferrara, and G. Scamarcio, "Experimental determination of the temperature distribution in trench-confined oxide vertical-cavity surface-emitting lasers," IEEE J. Quantum Electron. vol. 39, no. 6, pp. 701-707, Jun. 2003.

[8] M. Farzaneh et al., "Temperature profiling of VCSELs by thermoreflectance microscopy," IEEE Photon. Technol. Lett., vol. 19, no. 8, pp. 601-603, Apr. 2007.

[9] K. J. Greenberg, J. A. Summers, M. Farzaneh, and J. A. Hudgings, "Spatially-resolved thermal coupling in VCSEL arrays using thermoreflectance microscopy," in Proc. Conf. Lasers Electro-Opt., San Jose, CA, USA, 2008, pp. 1-2, doi: 10.1109/CLEO.2008.4551187.

[10] D. Pierścińska et al., "CCD thermoreflectance spectroscopy as a tool for thermal characterization of quantum cascade lasers," Semicond. Sci. Technol., vol. 31, no. 11, Sep. 2016, Art. no. 115006, doi: 10.1088/0268$1242 / 31 / 11 / 115006$

[11] S. Wang et al., "Time-resolved thermoreflectance imaging for mid-infrared quantum cascade laser," in Proc. Conf. Lasers Electro-Opt., Washington, DC, 2020, pp. 1-2, doi: 10.1364/CLEO_AT.2020.JTu2G.2.

[12] N. Becher et al., "Thermal imaging of buried heterostructure quantum cascade lasers (QCLs) and QCL arrays using CCD-based thermoreflectance microscopy," J. Appl. Phys., vol. 125, no. 3, Jan. 2019, Art. no. 033102, doi: 10.1063/1.5065507.

[13] R. McKenna et al., "Spatially resolved self-heating and thermal impedance of laser diodes using CCD-TR imaging," OSA Continuum, vol. 4, no. 4, pp. 1271-1281, Apr. 2021, doi: 10.1364/OSAC.422429.

[14] Q. Y. Lu et al., "Analysis of slot characteristics in slotted single-mode semiconductor lasers using the 2-D scattering matrix method," IEEE Photon. Technol. Lett., vol. 18, no. 24, pp. 2605-2607, Dec. 2006.

[15] Q. Lu, W. - H. Guo, D. Byrne, and J. F. Donegan, "Design of slotted singlemode lasers suitable for photonic integration," IEEE Photon. Technol. Lett., vol. 22, no. 11, pp. 787-789, Jun. 2010.

[16] F. Bello et al., "Traveling wave analysis for a high-order grating, partially slotted laser," IEEE J. Quantum Electron., vol. 51, no. 11, Nov. 2015, Art. no. 2200305.

[17] Q. Lu et al., "Single mode lasers based on slots suitable for photonic integration," Opt. Exp., vol. 19, no. 26, Dec. 2011, Art. no. B140, doi: 10.1364/OE.19.00B140.

[18] H. Wang and Y. Yu, "New theoretical model to analyze temperature distribution and influence of thermal transients of an SG-DBR laser," IEEE J. Quantum Electron., vol. 48, no. 2, pp. 107-113, Feb. 2012.

[19] S. B. Kuntze et al., "In situ resistance measurement of the p-type contact in InP-InGaAsP coolerless ridge waveguide lasers," Appl. Phys. Lett. vol. 86, no. 8, Feb. 2005, Art. no. 081111, doi: 10.1063/1.1869541

[20] M. Sotoodeh, A. H. Khalid, and A. A. Rezazadeh, "Empirical lowfield mobility model for III-V compounds applicable in device simulation codes," J. Appl. Phys., vol. 87, no. 6, pp. 2890-2900, Mar. 2000, doi: 10.1063/1.372274.

[21] D. Pierścińska et al., "High-resolution mirror temperature mapping in ganbased diode lasers by thermoreflectance spectroscopy," Jpn. J. Appl. Phys., vol. 56, no. 2, Jan. 2017, Art. no. 020302, doi: 10.7567/JJAP.56.020302.

[22] M. Farzaneh et al., "CCD-based thermoreflectance microscopy: Principles and applications," J. Phys. D, Appl. Phys., vol. 42, no. 14, Jul. 2009, Art. no. 143001, doi: 10.1088/0022-3727/42/14/143001.

[23] M. Farzaneh, D. Luerben, and J. A. Hudgings, "Thermal profiling of photonic integrated circuits by thermoreflectance microscopy," in Proc. Conf. Lasers Electro-Opt. Quantum Electron. Laser Sci. Conf., Long Beach, CA, USA, 2006, pp. 1-2, doi: 10.1109/CLEO.2006.4628240.

[24] D. Pierścińska, "Thermoreflectance spectroscopy_Analysis of thermal processes in semiconductor lasers," J. Phys. D: Appl. Phys., vol. 51, no. 1, Nov. 2018, Art. no. 013001, doi: 10.1088/1361-6463/aa9812.

[25] D. Luerssen, R. J. Ram, and J. A. Hudgings, "2-D thermal imaging of the optical power distribution in photonic integrated circuits," in Proc 17th Annu. Meeting IEEE Lasers Electro-Opt. Soc., 2004, pp. 163-164, doi: 10.1109/LEOS.2004.1363161.

[26] B. Vermeersch, J. Christofferson, K. Maize, A. Shakouri, and G. De Mey, "Time and frequency domain CCD-based thermoreflectance techniques for high-resolution transient thermal imaging," in Proc. 26th Annu. IEEE Semicond. Thermal Meas. Manage. Symp., Santa Clara, CA, 2010, pp. 228-234, doi: 10.1109/STHERM.2010.5444287.

[27] J. Christofferson et al., "Microscale and nanoscale thermal characterization techniques," in Proc. Int. Conf. Thermal Issues Emerg. Technol. Theory Application, 2007, pp. 3-9, doi: 10.1109/THETA.2007.363399.
Robert McKenna received the B.A. degree in nanoscience, physics, and chemistry of advanced materials from Trinity College Dublin, Ireland, in 2016. He is currently working toward the Ph.D. degree with the Semiconductor Photonics Group, Trinity College Dublin, Ireland. His current research interests include the design, characterisation and athermalisation of semiconductor laser diodes.

Simon Corbett received the B.A. degree in nanoscience, physics, and chemistry of advanced materials in 2016 from Trinity College Dublin, Ireland, where he is currently working toward the Ph.D. degree in physics under the supervision of Prof. David McCloskey. He is currently a Member of the Semiconductor Photonics Group with Trinity College Dublin. His current research interests include microscopic thermal imaging systems including CCD thermoreflectance imaging with a special focus on thermoelectric materials and devices.

Sepideh T. Naimi received the Ph.D. degree from Dublin City University, Dublin, Ireland, in 2016. After obtaining the Ph.D., she carried out an industrial position in Ireland to design the optical platforms of National Broadband Plan. In 2017, she joined Amazon Web Service as an Optical Engineer to design future amazon DWDM networks, and since 2018, she has been a Research Fellow with Semiconductor Photonics Group, Trinity College Dublin working on the design of energy-efficient laser devices for future optical networks.

Dovydas Mickus was born in Šiauliai, Lithuania, in 1997. He received the B.A. degree in experimental physics from Trinity College Dublin, Dublin, Ireland, in 2018. He is currently working toward the Ph. D. degree with the Semiconductor Photonics Group, Trinity College Dublin, Dublin, Ireland. His current research interests include the design, characterisation, and applications of semiconductor optoelectronic devices.

David McCloskey is currently an Assistant Professor of physics with Trinity College Dublin and an Investigator with the AMBER and CONNECT research centres, TCD. His research interests include nanoscale heating processes and heat transfer mechanisms in materials and devices. He is also working on improvements to the energy efficiency of solar panels.

John F. Donegan (Senior Member, IEEE) is currently a Professor of the physics and applications of light with Trinity College Dublin. He is the Principal Investigator with the AMBER Research Centre and the Deputy Director with the CRANN Nanoscience Centre, Trinity College Dublin. His research interests include photonic devices, including laser arrays for communications applications, device designs for heat-assisted magnetic recording and very recently on microresonators for broadband comb generation. 